\title{
Evidence-based recommender system and experimental validation for high-entropy alloys
}

\section{Minh-Quyet Ha} Japan Advanced Institute of Science and Technology https://orcid.org/0000-0003-4617-0059

\section{Nguyen-Duong Nguyen}

Japan Advanced Institute of Science and Technology

\section{Viet-Cuong Nguyen}

HPC SYSTEMS Inc.

\section{Takahiro Nagata}

National Institute for Materials Science

\section{Toyohiro Chikyow}

National Institute for Materials Science

\section{Hiori Kino}

National Institute for Materials Science

\section{Takashi Miyake}

National Institute of Advanced Industrial Science and Technology https://orcid.org/0000-0003-26583470

\section{Thierry Denœux}

Université de technologie de Compiègne https://orcid.org/0000-0002-0660-5436

\section{Van-Nam Huynh}

Japan Advanced Institute of Science and Technology

Hieu-Chi Dam ( $\nabla$ dam@jaist.ac.jp )

Japan Advanced Institute of Science and Technology https://orcid.org/0000-0001-8252-7719

\section{Article}

Keywords: Data-driven Approach, Element-combination Candidates, Descriptors, Evidence Theory, k-fold Cross-validation

Posted Date: March 1st, 2021

DOI: https://doi.org/10.21203/rs.3.rs-208862/v1

License: (c) (i) This work is licensed under a Creative Commons Attribution 4.0 International License. 
Version of Record: A version of this preprint was published at Nature Computational Science on July 19th, 2021. See the published version at https://doi.org/10.1038/s43588-021-00097-w. 


\title{
Evidence-based recommender system and experimental validation for high-entropy alloys
}

\author{
Minh-Quyet $\mathrm{Ha},{ }^{1}$ Duong-Nguyen Nguyen, ${ }^{1}$ Viet-Cuong Nguyen, ${ }^{2}$ Takahiro Nagata, ${ }^{3}$ Toyohiro Chikyow, ${ }^{4}$ Hiori \\ Kino, ${ }^{4}$ Takashi Miyake, ${ }^{5}$ Thierry Denœux, ${ }^{6}$ Van-Nam Huynh, ${ }^{1}$ and Hieu-Chi Dam ${ }^{1, a)}$ \\ 1) Japan Advanced Institute of Science and Technology, 1-1 Asahidai, Nomi, Ishikawa 923-1292, \\ Japan \\ ${ }^{2)}$ HPC SYSTEMS Inc., 3-9-15 Kaigan, Minato, Tokyo 108-0022, Japan \\ 3) Research Center for Functional Materials, National Institute for Materials Science, 1-2-1 Sengen, Tsukuba, \\ Ibaraki 305-0047, Japan \\ ${ }^{4)}$ Research and Services Division of Materials Data and Integrated System, National Institute for Materials Science, \\ 1-2-1 Sengen, Tsukuba, Ibaraki 305-004\%, Japan \\ ${ }^{5)}$ Research Center for Computational Design of Advanced Functional Materials, \\ National Institute of Advanced Industrial Science and Technology, 1-1-1 Umezono, Tsukuba, Ibaraki 305-8568, \\ Japan \\ 6) Université de technologie de Compiègne, CNRS, UMR 7253 Heudiasyc, Compiègne, \\ France
}

(Dated: 4 February 2021)

We present a data-driven approach to explore high-entropy alloys (HEAs). To overcome the challenges with numerous element-combination candidates, selecting appropriate descriptors, and the limitations and biased of existing data, we apply the evidence theory to develop a descriptor-free evidence-based recommender system (ERS) for recommending HEAs. The proposed system measures the similarities between element combinations and utilizes it to recommend potential HEAs. To evaluate the ERS, we compare its HEArecommendation capability with those of matrix-factorization- and supervised-learning-based recommender systems on four widely known data sets, including binary and ternary alloys. The results of experiments using $k$-fold cross-validation on the data sets show that the ERS outperforms all competitors. Furthermore, the ERS shows excellent extrapolation capabilities in experiments of recommending quaternary and quinary HEAs. We experimentally validate the most strongly recommended Fe-Co-based magnetic HEA, viz. FeCoMnNi, and confirm that it shows a body-centered cubic structure and is stable at high temperatures.

\section{INTRODUCTION}

Since their discovery, high-entropy alloys (HEAs), which comprise multiple elements and form highly disordered solid-solution phases, have attracted the interest of the scientific community owing to their promising properties and potential applications ${ }^{1,2}$. Such alloys show high strength-to-weight ratios, tensile strengths, and corrosion and oxidation resistances. Although the definition and element ratios of HEAs have not yet been established, we use the term "HEA" to refer to random alloys comprising multiple equiatomically combined elements and showing long-range periodic structures ${ }^{3-6}$. From the materials development perspective, specific element combinations that will most likely form single-phase HEAs must necessarily be recommended for experimental validation. Deductive and inductive approaches are both used to accomplish this task, and are based on entirely different concepts.

In the deductive approach, information about a particular element combination is used to predict whether it can form a single-phase HEA. Hence, it is necessary to understand the HEA formation mechanisms or begin with the quantum-mechanics equations derived based

\footnotetext{
a) Electronic mail: dam@jaist.ac.jp
}

on numerous first-principles calculations. In previous HEA research, it was hypothesized that HEA constituent elements form a single-phase solid solution owing to configurational-entropy-induced stabilization. However, this hypothesis is correct only for some multicomponent alloys, most of which have been experimentally demonstrated to form multiple phases ${ }^{7}$. Although much attention has been devoted to the formation mechanism driving HEA stability, the key factors governing the formation of single-phase HEAs remain unknown ${ }^{8}$. Constructing phase diagrams for multicomponent alloys by firstprinciples calculations can also directly predict which element combinations will form solid solutions ${ }^{9,10}$. However, this method involves energy calculations for many configurations and the implementation of statistical mechanical models for estimating thermodynamic properties, both of which are computationally demanding ${ }^{11}$. Therefore, it is imperative to search for HEAs by firstprinciples calculations.

In the inductive approach, information about the existing element combinations capable of forming singlephase HEAs is used to infer whether a particular combination of elements can form a single-phase HEA. Several screening methods have been developed using descriptors with parameters fitted to the available experimental data to predict the possible HEA element combinations ${ }^{12-14}$. Such methods require sufficient data to ensure prediction accuracy, which is unavailable for many combinations ${ }^{15}$. 
In addition, although it would be desirable to quantitatively evaluate the prediction uncertainty even if a high prediction accuracy cannot be obtained, this has not yet been achieved. Another challenge is that HEAs are very different from alloys composed of only one or two elements. Because new HEA phases are formed when more elements are added, it is very challenging to design material descriptors that can be typically used for alloys comprising different numbers of elements.

Therefore, we develop a system to recommend combinations of HEA elements without using any descriptors. We start by applying the evidence theory to learn the measure of similarity between combinations of elements from the data obtained for known multielement alloys by considering their HEA properties. We then apply the obtained similarities to recommend potential HEA candidates for element combinations. We used the evidence theory as it provides a suitable framework for handling incomplete and uncertain data. Therefore, the proposed approach can overcome the problem of inadequate experimental data that limits the inductive approach. In addition, using the evidence theory, we can use most of the information contained in the observed data and explicitly model the uncertainty involved in making subsequent inferences.

The proposed recommender system is based on the elemental substitution method widely used to synthesize various materials. This method is used to replace the element or group of elements with a counterpart showing similar chemical functions, such that the properties of the target material are not affected. The difficulty in this approach is the proper assessment of the similarity between the chemical functions of the alloy metal combinations to discover potential HEAs. To address this, we consider each pair of observed alloys as a piece of evidence to compare the contribution of their constituent elements or a combination thereof to the target property (i.e., HEA or multiphase formation).

Subsequently, we use the evidence theory ${ }^{16-20}$ to model and combine evidence on the similarity between the intended element combinations. The combined similarity evidence is then used to generate evidence for hypothesizing whether the substituted alloys are HEAs. Next, the evidence theory is applied again to model and combine the pieces of evidence on the properties of the substituted alloys. The ERS consists of three main steps:

1. Measure the similarity between element combinations: The evidence required for comparing the element combinations is obtained from the data, and all the pieces of evidence are combined to conclude the similarity between the element combinations.

2. Evaluate the hypothesis on the properties of the substituted alloys: The evidence for the substituted alloys is combined to evaluate the hypothesis about the target property (i.e., HEA or multiphase formation).
3. Rank substituted alloys: The substituted alloys are ranked according to various criteria based on the combined evidence of their target properties to recommend potential HEAs.

The details of the ERS will be explained in Section II. The recommendation capability of the proposed system is corroborated by both experimental and calculated data for multicomponent alloys ${ }^{5,21-24}$. The ERS, which does not require any material descriptors, obtained sufficient information to understand the HEA formation mechanisms. Moreover, the ERS recommended numerous alloys that can form solid solutions, which can be experimentally validated. Hence, the ERS can guide the design of HEAs with desirable features by selecting appropriate element combinations.

\section{METHODOLOGY}

We consider data set $\mathcal{D}$ consisting of $n$ alloys. $\mathcal{E}=$ $\left\{e_{1}, e_{2}, \ldots, e_{m}\right\}$ is the set of all $m$ componential elements that can be in $\mathcal{D}$. We assume that an alloy $A \in \mathcal{D}$ comprises $l$ equiatomically combined elements, $e_{A^{1}}, e_{A^{2}}, \cdots, e_{A^{l}} \in \mathcal{E}$. The alloy is then represented by a set of its components, $\left\{e_{A^{1}}, e_{A^{2}}, \cdots, e_{A^{l}}\right\}$. The property of interest $y_{A}$ of each alloy $A$ can be either $H E A$ or $\neg H E A$, which indicates whether $A$ is an HEA.

The evidence theory is applied by defining a frame of discernment ${ }^{18}$ containing all the possible values of the variable of interest. To evaluate the similarity between the element combinations, we define $\Omega_{\text {sim }}=$ $\{$ similar,dissimilar $\}$ as the frame of discernment. We then evaluate whether a particular $A$ forms an HEA, where $\Omega_{H E A}=\{H E A, \neg H E A\}$ is the frame of discernment.

\section{A. Measuring similarities between element combinations}

\section{Using mass functions to model evidence of similarities between element combinations}

Here, the solid-solution formability is discussed, along with the measure of similarity, in terms of substitutability between the elements. First, the evidence of similarities between the element combinations is evaluated. Each pair of alloys $A_{i}$ and $A_{j}$ in $\mathcal{D}$ is a source of evidence for measuring the substitutability between element combinations $C_{t}=A_{i}-\left(A_{i} \cap A_{j}\right)=A_{i}-A_{j}$ and $C_{v}=A_{j}-\left(A_{i} \cap A_{j}\right)=A_{j}-A_{i}$ (Figure 1 a). Here, we only consider the evidence obtained from nondisjoint pairs $A_{i}$ and $A_{j}$. The nonempty intersection set $A_{i} \cap A_{j}$ is considered as the context for the similarity measurement. The difference in the solid-solution formability (i.e., $H E A$ or $\neg H E A$ ) of alloys $A_{i}$ and $A_{j}$ is used to quantify the hypothesis of the similarity between $C_{t}$ and 


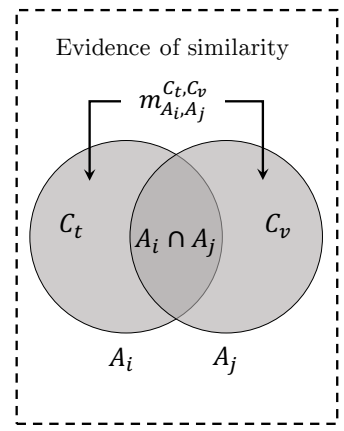

(a)

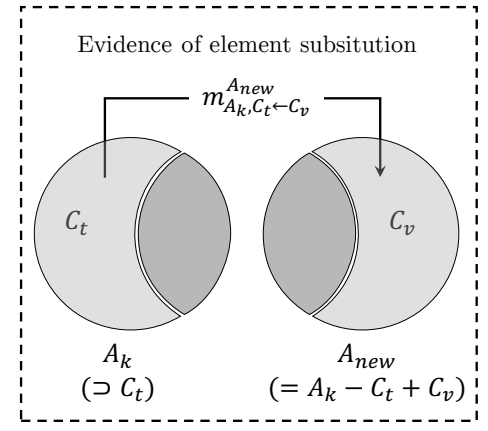

(b)
FIG. 1. Schemes for using mass functions to model evidence of (a) similarities between element combinations and (b) new alloys by element substitution.

$C_{v}$. If $y_{A_{i}}=y_{A_{j}}$, then $C_{t}$ and $C_{v}$ are substitutable, while if $y_{A_{i}} \neq y_{A_{j}}$, then $C_{t}$ and $C_{v}$ are not substitutable.

Hence, within the framework of the evidence theory, we represent the frame of discernment ${ }^{18,19}$ for the similarity between element combinations $C_{t}$ and $C_{v}$ by $\Omega_{\text {sim }}=\{$ similar, dissimilar $\}$. The evidence collected from alloys $A_{i}$ and $A_{j}$ is then represented by a mass function $^{18,19}$ (or a basic probability assignment), $m_{A_{i}, A_{j}}^{C_{t}, C_{v}}$, which assigns probability masses to all the nonempty subsets of $\Omega_{\text {sim }}$ (i.e., $\{$ similar $\},\{$ dissimilar $\}$, and $\{$ similar, dissimilar $\})$, as follows:

$$
\begin{gathered}
m_{A_{i}, A_{j}}^{C_{t}, C_{v}}(\{\text { similar }\})= \begin{cases}\alpha & \text { if } y_{A_{i}}=y_{A_{j}} \\
0 & \text { otherwise }\end{cases} \\
m_{A_{i}, A_{j}}^{C_{t}, C_{v}}(\{\text { dissimilar }\})=\left\{\begin{array}{ll}
\alpha & \text { if } y_{A_{i}} \neq y_{A_{j}} \\
0 & \text { otherwise }
\end{array},\right. \\
m_{A_{i}, A_{j}}^{C_{t}, C_{v}}(\{\text { similar, dissimilar }\})=1-\alpha
\end{gathered}
$$

Note that the masses assigned to \{similar $\}$ and $\{$ dissimilar $\}$ indicate the degrees of belief exactly committed to $A_{i}$ and $A_{j}$ to support the similarity and dissimilarity between $C_{t}$ and $C_{v}$, respectively. The weight assigned to subset $\{$ similar, dissimilar $\}$ expresses the degree of belief that $A_{i}$ and $A_{j}$ provide no information about the similarity (or dissimilarity) between $C_{t}$ and $C_{v}$. The sum of the masses assigned to all three nonempty subsets of $\Omega_{\text {sim }}$ is 1 . Hence, $\alpha$ is the only parameter in this model and is determined by a thorough search for the best cross-validation score for each data set (Section S III of Supplementary materials).

\section{Combining multiple pieces of evidence about the similarity between elements}

We assume that we can collect $l$ pieces of evidence from $\mathcal{D}$ to compare a specific pair of element combina- tions, $C_{t}$ and $C_{v}$. Each piece of evidence is modeled by the mass function $m_{\text {evidence }}^{C_{t}, C_{v}}$ (Subsection II A 1), which assigns probability masses to all three nonempty subsets, $\{$ similar $\},\{$ dissimilar $\}$, and $\{$ similar,dissimilar $\}$. If no evidence is found, the mass function $m_{n o n e}^{C_{t}}, C_{v}$ is initialized, which assigns a probability mass of 1 to subset $\{$ similar, dissimilar $\} . m_{n o n e}^{C_{t}, C_{v}}$ models the condition under which no information about the similarity (or dissimilarity) between $C_{t}$ and $C_{v}$ is available. Any two pieces of evidence $a$ and $b$ modeled by the corresponding mass functions $m_{a}^{C_{t}}, C_{v}$ and $m_{b}^{C_{t}, C_{v}}$ can be combined using the Dempster rule ${ }^{16}$ to assign the joint mass $m_{a, b}^{C_{t}, C_{v}}$ to each subset $\omega$ of $\Omega_{\text {sim }}$ (i.e. $\{$ similar $\},\{$ dissimilar $\}$, or $\{$ similar, dissimilar $\})$ as follows:

$$
\begin{aligned}
m_{a, b}^{C_{t}, C_{v}}(\omega) & =\left(m_{a}^{C_{t}, C_{v}} \oplus m_{b}^{C_{t}, C_{v}}\right)(\omega) \\
& =\frac{\sum_{\forall \omega_{k} \cap \omega_{h}=\omega} m_{a}^{C_{t}, C_{v}}\left(\omega_{k}\right) \times m_{b}^{C_{t}, C_{v}}\left(\omega_{h}\right)}{1-\sum_{\forall \omega_{k} \cap \omega_{h}=\emptyset} m_{a}^{C_{t}, C_{v}}\left(\omega_{k}\right) \times m_{b}^{C_{t}, C_{v}}\left(\omega_{h}\right)},
\end{aligned}
$$

where $\left\{\omega_{k}, \omega_{h}\right\}$ are arbitrary sets of subsets of $\Omega_{\text {sim }}$. Note that the Dempster rule is commutative and yields the same result by changing the order of $m_{a}^{C_{t}}, C_{v}$ and $m_{b}^{C_{t}}, C_{v}$. All the obtained $l$ mass functions corresponding to the collected $l$ pieces of evidence from $\mathcal{D}$ are then combined using the Dempster rule to assign the final mass $m_{\mathcal{D}}^{C_{t}, C_{v}}$ as follows:

$$
m_{\mathcal{D}}^{C_{t}, C_{v}}(\omega)=\left(m_{1}^{C_{t}, C_{v}} \oplus m_{2}^{C_{t}, C_{v}} \oplus \cdots \oplus m_{l}^{C_{t}, C_{v}}\right)(\omega) .
$$

Similar analyses are performed for all pairs of element combinations of interest to obtain a matrix $M$ consisting of all similarities between them.

\section{B. Recommending potential HEAs}

\section{Modeling evidence of potential HEAs for substituted alloys}

Using the substitution method, we replace a combination of elements, $C_{t}$, in an existing alloy, $A_{k},\left(C_{t} \subset A_{k}\right)$ with a combination of elements, $C_{v}$, adequate to obtain alloy $A_{\text {new }}$ showing a property (label $y_{A_{\text {new }}}$ ) similar to that of $A_{k}$ (label $y_{A_{k}}$ ). On the basis of the label of $A_{k}$ and the similarity between $C_{t}$ and $C_{v}$, the basic beliefs on the label of $A_{n e w}$ are quantified (Figure $1 \mathrm{~b}$ ). If $C_{t}$ and $C_{v}$ are substitutable (non-substitutable), this serves as a piece of evidence that the labels of $A_{\text {new }}$ and $A_{k}$ are the same(different). Since $y_{A_{k}}$ is already known, the evidence collected from $A_{k}, C_{t}$, and $C_{v}$ is also evidence of the label of $A_{n e w}$.

From the evidence theory, the evidence collected from $A_{k}, C_{t}$, and $C_{v}$ is then represented by the mass function $m_{A_{k}, C_{t} \leftarrow C_{v}}^{A_{n e w}}$, which assigns probability masses to all the 
TABLE I. Summary of alloy data sets used in evaluation experiments. Columns show names and numbers of alloys, HEAs contained in each data set, and number of all possible corresponding alloy candidates.

\begin{tabular}{|c|c|c|c|c|c|}
\hline Data set & \#alloys & \#HEAs & \#candidates & HEAs rate & Observation rate \\
\hline $\mathcal{D}_{\text {ASMI16 }}{ }^{21}$ & 45 binary alloys & 45 & 351 & $100 \%$ & $13 \%$ \\
\hline $\mathcal{D}_{\text {CALPHAD }} 5,22$ & 243 ternary alloys & 243 & 2925 & $100 \%$ & $9 \%$ \\
\hline $\mathcal{D}_{\text {AFLOW }}{ }^{23}$ & $\begin{array}{l}117 \text { binary alloys } \\
441 \text { ternary alloys }\end{array}$ & $\begin{array}{c}60 \\
234\end{array}$ & $\begin{array}{c}351 \\
2925\end{array}$ & $\begin{array}{l}51 \% \\
53 \%\end{array}$ & $\begin{array}{l}33 \% \\
15 \%\end{array}$ \\
\hline $\mathcal{D}_{\text {LTVC }}{ }^{24}$ & $\begin{array}{l}117 \text { binary alloys } \\
441 \text { ternary alloys }\end{array}$ & $\begin{array}{c}58 \\
148\end{array}$ & $\begin{array}{c}351 \\
2925\end{array}$ & $\begin{array}{l}49 \% \\
33 \%\end{array}$ & $\begin{array}{l}33 \% \\
15 \%\end{array}$ \\
\hline $\begin{array}{l}\mathcal{D}_{\text {AFLOWW }}^{\text {quaternary 23 }} \\
\mathcal{D}_{\text {LTVC }}^{\text {quaternary 24 }}\end{array}$ & $\begin{array}{l}1,110 \text { quaternary alloys } \\
1,110 \text { quaternary alloys }\end{array}$ & $\begin{array}{l}754 \\
480\end{array}$ & $\begin{array}{l}17,550 \\
17,550\end{array}$ & $\begin{array}{l}68 \% \\
43 \%\end{array}$ & $\begin{array}{l}6 \% \\
6 \%\end{array}$ \\
\hline $\begin{array}{l}\mathcal{D}_{\text {AFLOW }}^{\text {quinary } 23} \\
\mathcal{D}_{\text {LTVC }}^{\text {quinary 24 }}\end{array}$ & $\begin{array}{l}130 \text { quinary alloys } \\
130 \text { quinary alloys }\end{array}$ & $\begin{array}{c}129 \\
91\end{array}$ & $\begin{array}{l}80,730 \\
80,730\end{array}$ & $\begin{array}{l}99 \% \\
70 \%\end{array}$ & $\begin{array}{l}0.16 \% \\
0.16 \%\end{array}$ \\
\hline
\end{tabular}

nonempty subsets of $\Omega_{H E A}$ (i.e., $\{H E A\},\{\neg H E A\}$, and $\{H E A, \neg H E A\})$, as follows:

$$
\begin{gathered}
m_{A_{k}, C_{t} \leftarrow C_{v}}^{A_{n e w}}(\{H E A\})= \begin{cases}s\left(C_{t}, C_{v}\right) & \text { if } y_{A_{k}}=H E A, \\
0 & \text { otherwise }\end{cases} \\
m_{A_{k}, C_{t} \leftarrow C_{v}}^{A_{n e w}}(\{\neg H E A\})=\left\{\begin{array}{ll}
s\left(C_{t}, C_{v}\right) & \text { if } y_{A_{k}}=\neg H E A \\
0 & \text { otherwise }
\end{array},\right. \\
m_{A_{k}, C_{t} \leftarrow C_{v}}^{A_{n e w}}(\{H E A, \neg H E A\})=1-s\left(C_{t}, C_{v}\right),
\end{gathered}
$$

where $s\left(C_{t}, C_{v}\right)=m_{\mathcal{D}}^{C_{t}, C_{v}}(\{$ similar $\})$ indicates the exact degree of belief committed to evidence from $\mathcal{D}$ to support the similarity between $C_{t}$ and $C_{v}$.

Note that the masses assigned to $\{H E A\}$ and $\{\neg H E A\}$ reflect the levels of confidence whereby $A_{k}$ and the substitution of $C_{v}$ for $C_{t}$ support the probabilities that $A_{n e w}$ is or is not an HEA, respectively. The mass assigned to subset $\{H E A, \neg H E A\}$, expresses the probability that $A_{k}, C_{t}$, and $C_{v}$ provide no information about the property of $A_{\text {new }}$. The sum of the probability masses assigned to all three nonempty subsets of $\Omega_{H E A}$ is 1 .

\section{Combining multiple pieces of evidence for recommending potential HEAs}

Multiple pieces of evidence about the label of each new alloy are combined using the same method as described in Subsection II A 2. We assume that for a specific hypothetical alloy, $A_{n e w}$, we can collect $l$ pieces of evidence about its properties from $\mathcal{D}$ (pair of $A_{\text {host }}$ and the corresponding substitution to obtain $A_{\text {new }}$ from $A_{\text {host }}$ ). Each piece of evidence is modeled using mass function $m_{\text {evidence }}^{A_{\text {new }}}$ (Subsection II B 1), which assigns probability masses to all three nonempty subsets $\{H E A\},\{\neg H E A\}$, and $\{H E A, \neg H E A\}$. If no evidence is found, $m_{\text {none }}^{A_{\text {new }}}$ is initialized and a probability mass of 1 is applied to set $\{H E A, \neg H E A\} . m_{n o n e}^{A_{\text {new }}}$ models the condition that no information about the label of $A_{\text {new }}$ can be obtained from $\mathcal{D}$. The $l$ obtained mass functions for $A_{\text {new }}$ are then combined using the Dempster rule ${ }^{16}$ to assign a final mass $m_{\mathcal{D}}^{A_{\text {new }}}$ to each subset $\omega$ of $\Omega_{H E A}$.

Similar analyses are performed for all $A_{\text {new }}$. Finally, we use the obtained mass functions to rank the alloys and recommend potential HEAs, where the alloys with the highest $m^{A_{\text {new }}}(\{H E A\})$ values have the greatest potential of being HEAs. The entire data analysis flow is shown in Figure S 2 of the Supplementary material.

\section{EXPERIMENTS, RESULTS, AND DISCUSSION}

\section{A. Experimental settings}

\section{Alloy data sets}

We use eight data sets (Table I) consisting of binary, ternary, quaternary, and quinary alloys comprising multiple equiatomically combined elements to evaluate the proposed system for recommending HEAs and revealing the HEA formation mechanisms. The alloys contained in the data sets comprise $\mathcal{E}=\{\mathrm{Fe}, \mathrm{Co}, \mathrm{Ir}, \mathrm{Cu}, \mathrm{Ni}, \mathrm{Pt}$, $\mathrm{Pd}, \mathrm{Rh}, \mathrm{Au}, \mathrm{Ag}, \mathrm{Ru}, \mathrm{Os}, \mathrm{Si}, \mathrm{As}, \mathrm{Al}, \mathrm{Tc}, \mathrm{Re}, \mathrm{Mn}, \mathrm{Ta}, \mathrm{Ti}$, $\mathrm{W}, \mathrm{Mo}, \mathrm{Cr}, \mathrm{V}, \mathrm{Hf}, \mathrm{Nb}$, and $\mathrm{Zr}$. Any alloy contained in the following data sets is predicted as an HEA if its order-disorder transition temperature is below its melting temperature. All the data sets are shown in detail in Section S I of the Supplementary material. 


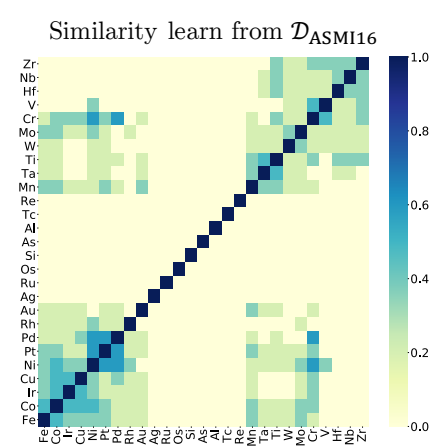

(a)

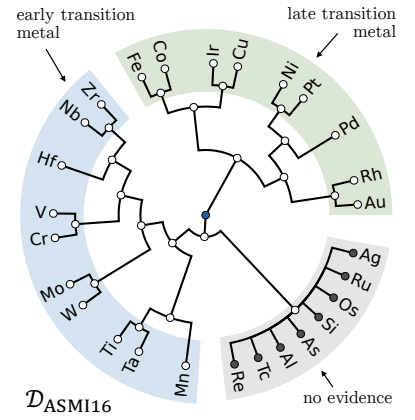

(e)

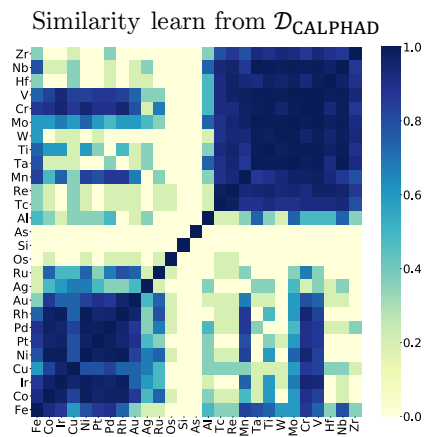

(b)

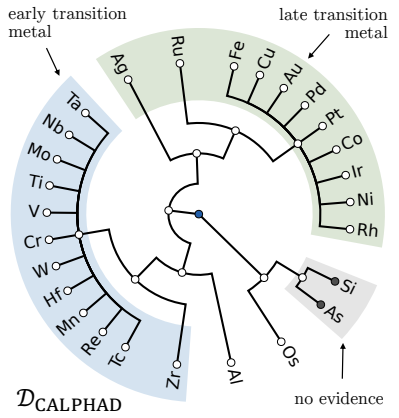

(f)

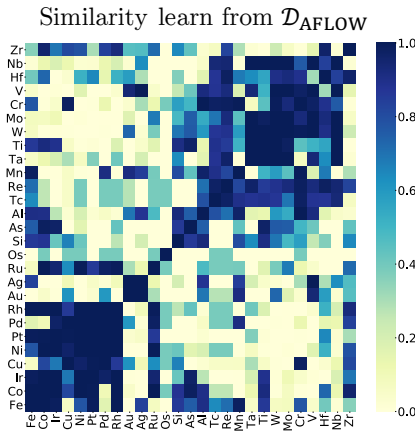

(c)

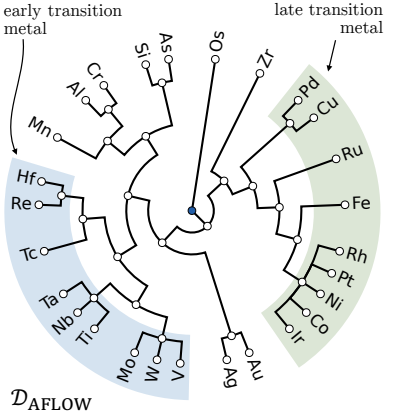

(g)
Similarity learn from $\mathcal{D}_{\text {LTVC }}$

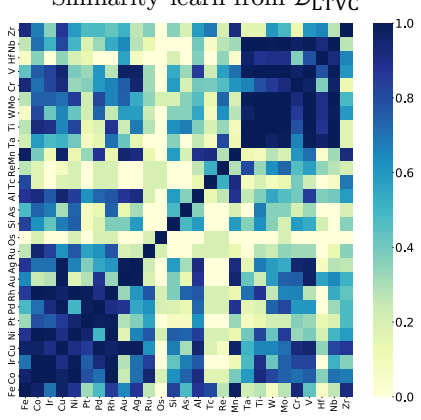

(d)

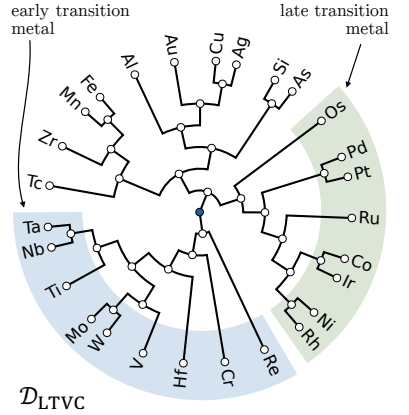

(h)

FIG. 2. Top: Heat maps for similarity matrices among 27 elements $\mathcal{E}$ obtained from $\mathcal{D}_{\mathrm{ASMI} 16}, \mathcal{D}_{\mathrm{CALPHAD}}, \mathcal{D}_{\mathrm{AFLOW}}$, and $\mathcal{D}_{\text {LTVC }}$ data sets. Center: Hierarchically clustered structures of all elements in $\mathcal{E}$ constructed using these similarity matrices and hierarchical agglomerative clustering. The blue, green, and gray regions indicate groups of early and late transition metals, and elements without similarity evidence, respectively.

\section{Comparison of HEA-recommendation performances of various systems}

We compare the HEA-recommendation performance of the proposed ERS with those of matrix-based recommender systems ${ }^{25}$ previously developed using nonnegative matrix factorization $(\mathrm{NMF})^{26}$ and singular-value decomposition (SVD) $)^{27}$. To use the matrix-based recommender systems for exploring potential HEAs, we apply rating-matrix representations. In addition, the performances of recommender systems based on supervisedlearning $\left(\mathrm{SVM}^{28}\right.$, logistic-regression ${ }^{29}$, decision-tree ${ }^{30}$, and Naïve-Bayes ${ }^{31}$ ) methods are compared with that of the ERS. We apply a compositional descriptor to employ the SVM- and logistic-regression-based recommender systems. The binary elemental descriptor is used to represent the alloys in our system and in the decisiontree and Naïve-Bayes-based recommender systems. The material descriptors are shown in detail in the Supplementary material (Section S II).

\section{B. Learning similarities between material components obtained from alloy data sets}

By applying the proposed ERS to the $\mathcal{D}_{\text {ASMI16, }}$ $\mathcal{D}_{\text {CALPhAD }}, \mathcal{D}_{\text {AflOW }}$, and $\mathcal{D}_{\text {LTVC }}$ data sets, we assess the similarities between the $\mathcal{E}$ elements and all the possible binary combinations obtained therein. Figures 2 (a, $\mathrm{b}$, c, and d) show the $M_{\mathrm{ASMI} 16}, M_{\mathrm{CALPHAD}}, M_{\mathrm{AFLOW}}$, and $M_{\text {LTVC }}$ similarity matrices obtained for all the $\mathcal{E}$ elements in the first four experiments. Each matrix element is the probability mass that the similarity mass function of the corresponding element pair is assigned to subset $\{$ similar $\}$ of $\Omega_{\text {sim }}$. These matrix elements indicate the degree of belief learned from the similarity data of the corresponding element pairs. These similarity matrices are then properly transformed into distance matrices to which hierarchical agglomerative clustering ${ }^{32}$ can be applied to construct the corresponding hierarchically clustered structures of all the $\mathcal{E}$ elements (Figure $2 \mathrm{e}, \mathrm{f}, \mathrm{g}$, and $\mathrm{h}$ ).

The similarity matrix learned from $\mathcal{D}_{\text {ASMI16 }}$ reveals three distinct element groups (Figure 2 e) consisting of Ti, V, Cr, Mn, Zr, Nb, Mo, Hf, Ta, and W; Fe, Co, Ni, $\mathrm{Cu}, \mathrm{Rh}, \mathrm{Pd}$, Ir, Pt, and $\mathrm{Au}$; and $\mathrm{Al}, \mathrm{Ag}, \mathrm{Tc}, \mathrm{Si}, \mathrm{Ru}$, As, Re, and Os, where the first two groups correspond to the early and late transition metals, respectively. Given the similar physical and chemical properties of these elements, the high degree of similarity between the elements within the same group, as revealed by the ERS, is rational. Interestingly, the obtained similarity matrix shows a remarkable similarity between Mn (an earlier transition metal) and $\mathrm{Au}$ (a late transition metal). 


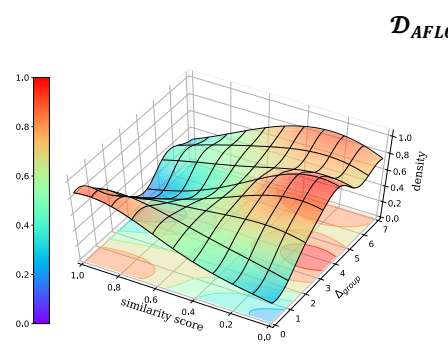

(a)
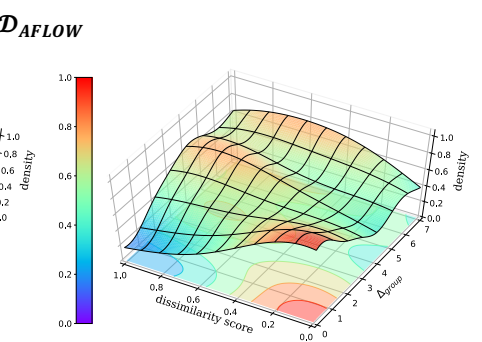

(b)

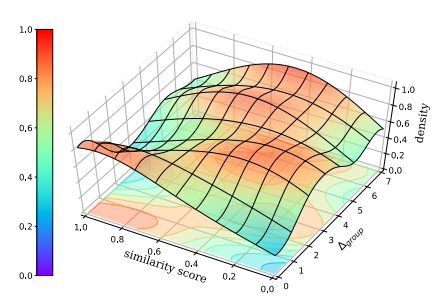

(c)
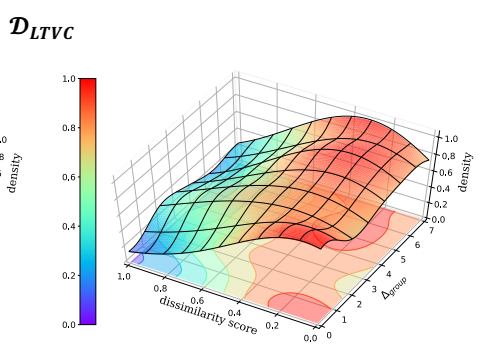

(d)

FIG. 3. Correlation between pairwise similarity and difference in group index $\left(\Delta_{\text {group }}\right)$ for every element pair in $\mathcal{E}$, as learned from both data sets: $\mathcal{D}_{\text {AfLOW }}(\mathrm{i}, \mathrm{j})$ and $\mathcal{D}_{\text {LTVC }}(\mathrm{k}, \mathrm{l})$.

The similarity matrix obtained from $\mathcal{D}_{\text {ASMI16 }}$ shows none of the belief about the similarities among the elements in the third group and between the elements of the third group and the other two groups because the binary alloys contained in $\mathcal{D}_{\mathrm{ASMI}}$ do not contain these elements (Figure S 1 a). Therefore, no evidence of similarities can be collected from $\mathcal{D}_{\text {ASMI16 }}$ for these elements.

The similarity matrix learned from $\mathcal{D}_{\text {CALPHAD }}$ also reveals three distinct element groups. Compared to the three groups obtained from $\mathcal{D}_{\text {ASMI16 }}$, those obtained from $\mathcal{D}_{\text {CALPHAD }}$ reveal three somewhat modified element groups (Figure $2 \mathrm{f}$ ). Because $\mathcal{D}_{\mathrm{CALPHAD}}$ contains some Tc- and Re-containing alloys, these elements join the group of early transition metals. Similarly, $\mathcal{D}_{\mathrm{CALPHAD}}$ contains more $\mathrm{Ag}$ - and Au-containing alloys, and these elements join the group of late transition metals. Therefore, only $\mathrm{Al}, \mathrm{Si}, \mathrm{As}$, and Os remain in the third group. Although no evidence of any similarities between Si and As can be collected from $\mathcal{D}_{\text {CALPHAD }}$ (Figure S 1 a), Os and $\mathrm{Al}$ are somewhat similar to the group of late transition metals and the first and second groups, respectively.

In contrast to the previously obtained results, it is difficult to divide all the elements contained in $\mathcal{E}$ into groups according to the similarity matrix learned from $\mathcal{D}_{\text {AFLOW }}$. However, some characteristic groups of metallic elements are distinct. Although two distinct groups of early or late transition metals are observed(Figure $2 \mathrm{~g}$ ), there are some notable differences between these results and those obtained from $\mathcal{D}_{\text {ASMI16 }}$ and $\mathcal{D}_{\text {CALPHAD }}$ (Section S IV ). In addition, the similarity matrix learned from $\mathcal{D}_{\text {AFLOW }}$ does not show any similarity between Os and any of the other elements because very few Os-containing alloys are contained in the data set (Figure S 1 a).

Furthermore, the result of the experiment with $\mathcal{D}_{\text {LTVC }}$ shows that the similarity matrices learned from $\mathcal{D}_{\text {LTVC }}$ and $\mathcal{D}_{\text {AFLOW }}$ are approximately similar. However, the hierarchically clustered structure constructed from $\mathcal{D}_{\text {LTVC }}$ indicates that $\mathrm{Cu}, \mathrm{Ag}$, and $\mathrm{Au}$ form a distinct subgroup (Figure $2 \mathrm{~h}$ ).

Figure 3 shows the correlation between the pairwise similarities learned from the $\mathcal{D}_{\text {AFLOW }}$ and $\mathcal{D}_{\text {LTVC }}$ data sets and the corresponding difference between the periodic-table group index obtained for each of the ele- ment pairs contained in $\mathcal{E}$. Clearly, the elements showing the same periodic-table group index $\left(\Delta_{\text {group }}=0\right)$ tend to show high similarity scores (Figure 3 a and c) and low dissimilarity scores (Figure $3 \mathrm{~b}$ and d), indicating that an element is the most similar to those in the same or next group in the periodic table. Therefore, the elements in the same group similarly contribute to HEA formation and are substitutable for each other. However, it should be noted that several pairs of elements (i.e., Ti and $\mathrm{Zr}$, $\mathrm{Cu}$ and $\mathrm{Ag}$, and $\mathrm{Fe}$ and $\mathrm{Ru}$; and $\mathrm{Ti}$ and $\mathrm{Zr}, \mathrm{Mn}$ and $\mathrm{Re}$, and $\mathrm{Ni}$ and $\mathrm{Pd}$ contained in $\mathcal{D}_{\text {AFLOW }}$ and $\left.\mathcal{D}_{\text {LTVC }}\right)$, as shown in Figure $2 \mathrm{c}$ and Figure $2 \mathrm{~d}$, respectively, have a similarity with a low degree of belief even though the elements belong to the same groups.

Furthermore, as the difference in the group index increases from 0 to 4 , the similarity between the elements decreases $\left(\Delta_{\text {group }}: 0 \rightarrow 4\right)$. The results learned from the $\mathcal{D}_{\text {AFLOW }}$ and $\mathcal{D}_{\text {LTVC }}$ data sets both show that the elements are the least similar when the difference between their group indices is three or four. However, the elements become slightly more similar as $\Delta_{\text {group }}$ increases from 5 to 7 , which is consistent with the domain knowledge about the differences between early and late transition metals.

\section{Evaluation of HEA-recommendation capability by cross-validation}

We apply $k$-fold cross-validation to the $\mathcal{D}_{\text {ASMI16, }}$, $\mathcal{D}_{\text {CALPHAD }}, \mathcal{D}_{\text {AFLOW }}$, and $\mathcal{D}_{\text {LTVC }}$ data sets to assess the HEA-recommendation capabilities of the ERS, the four matrix-based recommender systems ${ }^{25}$, and the four supervised-learning-method-based (i.e., decisiontree, Naïve-Bayes, logistic-regression, and SVM) recommender systems.

All the alloys contained in each data set are divided into $k$ partitions by random sampling. We use $k-1$ partitions (i.e., the training set) for learning the corresponding similarity matrix, and the one remaining partition for testing (i.e., the test set). The learned similarity matrix is then used to rank all the possible combinatorial alloys other than those used to train the similarity matrix. All 


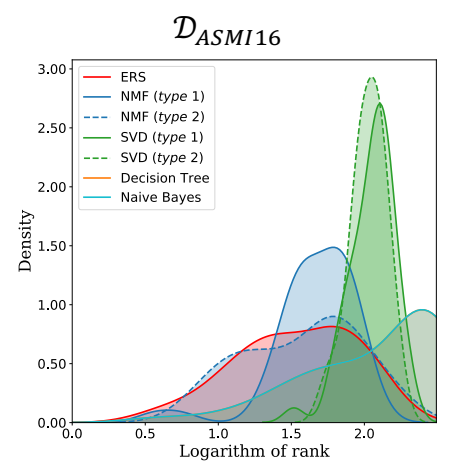

(a)

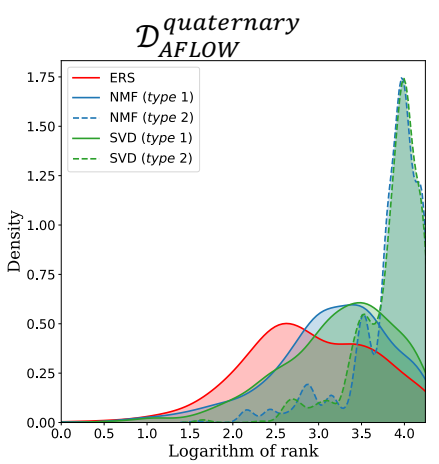

(e)

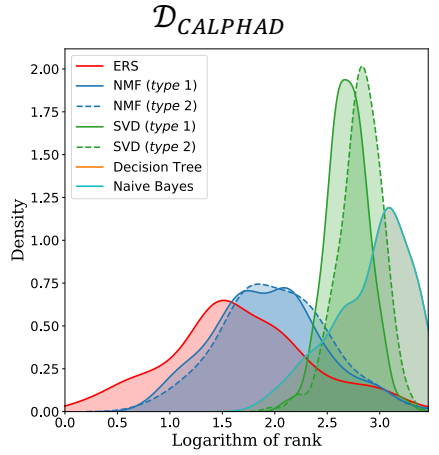

(b)

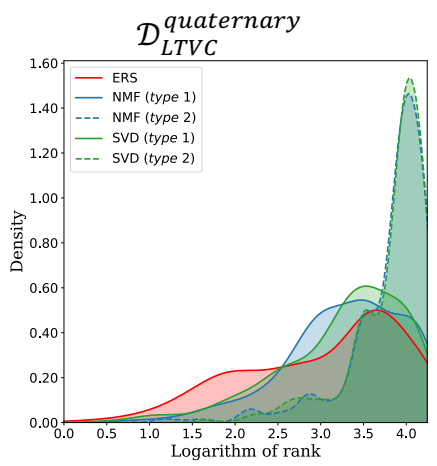

(f)

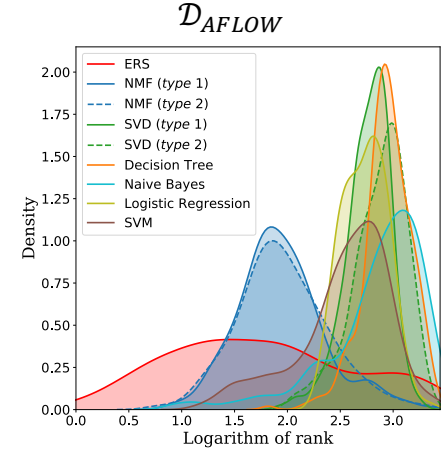

(c)

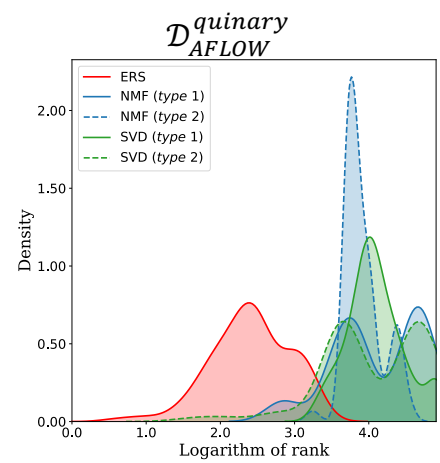

(g)

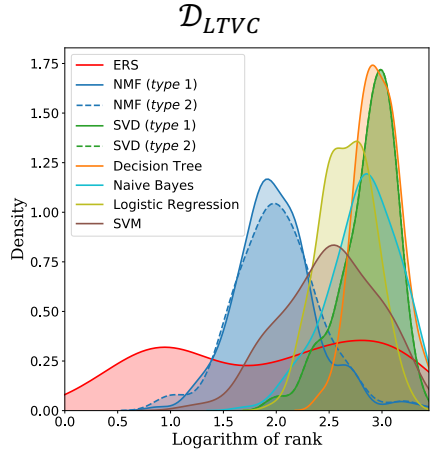

(d)

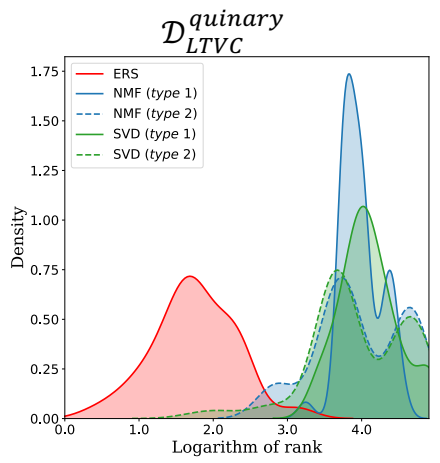

(h)

FIG. 4. Probability density functions of the rank of the HEAs in the test sets in $\mathcal{D}_{\mathrm{ASmi16}}, \mathcal{D}_{\mathrm{CALPHAD}}, \mathcal{D}_{\mathrm{AFLOW}}, \mathcal{D}_{\mathrm{LTVC}}$, $\mathcal{D}_{\text {AFLOW }}^{\text {quaternary }}, \mathcal{D}_{\text {LTVC }}^{\text {quaternary }}, \mathcal{D}_{\text {AFLOW }}^{\text {quinary }}$, and $\mathcal{D}_{\text {LTVC }}^{\text {quinary }}$ experiments. The ranks of HEAs in the test sets are expressed on a base- 10 logarithmic scale.

the alloys contained in the remaining data partitions not used for learning the similarity matrix are also ranked. The resulting alloy rankings are then used to evaluate the HEA-recommendation performance. We designed a virtual experiment that sequentially identifies the alloys on the basis of the order in which they were previously ranked. To evaluate the HEA-recommendation capability of the proposed ERS, we monitor the rank of HEAs in the test set and the HEA recall depending on the number of trials required to identify all possible HEAs. Note that all alloys contained in the $\mathcal{D}_{\text {ASMI16 }}$ and $\mathcal{D}_{\text {CALPHAD }}$ data sets are confirmed HEAs; the alloys labeled $\neg H E A$ are not contained in these two data sets. In contrast, $\mathcal{D}_{\text {AFLOW }}$ and $\mathcal{D}_{\text {LTVC }}$ contain both $H E A$ - and $\neg H E A$ labeled alloys. Only the $H E A$-labeled alloys in the test set are used for the evaluation. The experimental conditions are shown in detail in the Supplementary material (Section S V A).

Figures $4(\mathrm{a}-\mathrm{d})$ illustrate the distributions of the HEA ranks of the test set recommended by the different systems. The HEAs in the test set are generally recommended with higher rank using the ERS (i.e., the ERS rank distributions are on the left of the curves for the other systems). Consequently, the ERS can significantly reduce the number of trials required to recover the HEAs in the test set compared to the competitor systems. Only in the experiment with $\mathcal{D}_{\mathrm{ASMI}}$, the distributions of the rank using the ERS and NMF (type 2) are somewhat similar (Fig. 4 a). We also monitor the dependence of the HEA recall ratio on the number of trials required to measure the HEA-recommendation performance of the ERS quantitatively. The results are listed in detail in the Supplementary material (Section S VB). In summary, ERS outperforms the other systems in recalling one-half and three-quarters of the HEAs in the test set. However, the ERS cannot reliably recall the remaining one-quarter of the HEAs because insufficient evidence is available in the training data to make inferences about the remaining HEAs. Interestingly, in the $\mathcal{D}_{\mathrm{ASMI} 16}$ and $\mathcal{D}_{\mathrm{CALPHAD}}$ experiments, the supervised-method-based recommender systems either approximately randomly selected possible HEAs (Naïve Bayes and decision tree) or could not rank any at all (logistic regression and SVM) because these data sets contain only positively labeled HEAs.

\section{Evaluation of HEA-recommendation capability by extrapolation}

It should be noted that when extrapolating the number of components, the recommender systems based on supervised learning methods $\left(\mathrm{SVMs}^{28}{ }^{2}\right.$ logistic regression ${ }^{29}$, 


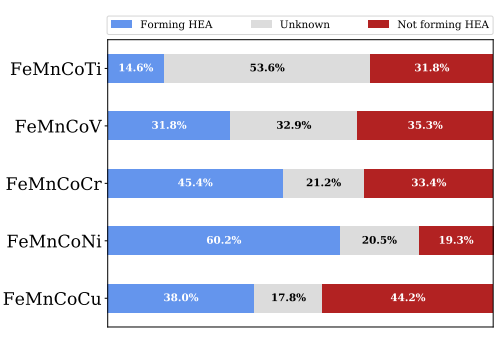

(a)

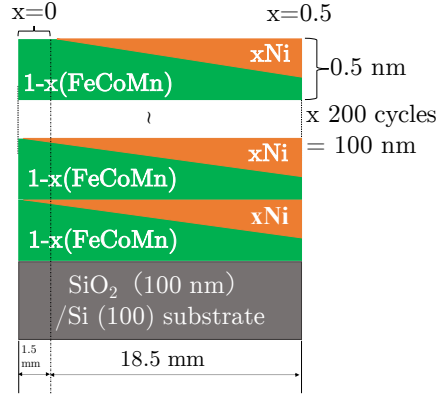

(b)

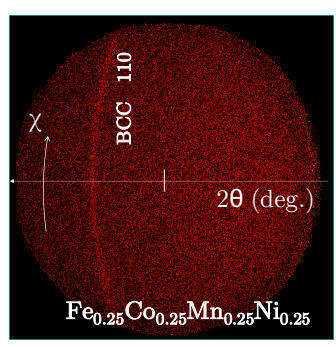

(c)

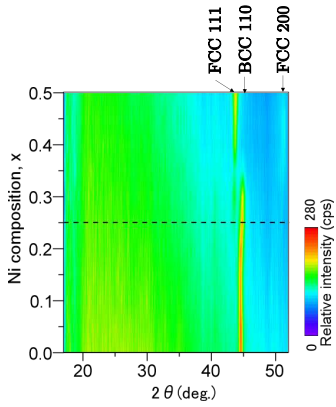

(d)

FIG. 5. (a) Recommended candidates for Fe-Co-based HEAs containing first-transition-series elements: FeMnCoTi, FeMnCoV, FeMnCoCr, FeMnCoNi, and FeMnCoCu. (b) Schematic illustration of the sample. (c) 2D-XRD image of $\mathrm{Fe}_{0.25} \mathrm{Co}_{0.25} \mathrm{Mn}_{0.25} \mathrm{Ni}_{0.25}$ film. (d) Heat map of $\omega-2 \theta$ scan converted from 2D-XRD images of $1-x(\mathrm{FeCoMn})-x \mathrm{Ni}$ films prepared with various $\mathrm{Ni}$ contents.

decision trees ${ }^{30}$, and Naïve Bayes ${ }^{31}$ ) cannot be applied, as shown in the Supplementary materials (Section S II). Therefore, to evaluate the HEA-recommendation capability by extrapolation, we compare the performances of the ERS and matrix-based recommender systems in experiments with $\mathcal{D}_{\text {AFLOW }}^{\text {quaternary }}, \mathcal{D}_{\text {AFLOW }}^{\text {quinary }}, \mathcal{D}_{\text {LTVC }}^{\text {quaternary }}$, and $\mathcal{D}_{\text {LTVC }}^{\text {quinary }}$ data sets. The settings of the experiments are shown in detail in the Supplementary material (Section S VIA).

Figures $4(\mathrm{e}-\mathrm{h})$ illustrate the distributions of the recommended HEA rank of the quaternary and quinary HEAs in the test set that are extrapolated using recommender systems. The obtained results show that the ERS outperforms the capability of the competitor systems for recommending quaternary HEAs (Fig. 4 e and f) and drastically outperforms the capability of the other systems for recommending quinary HEAs (Fig. $4 \mathrm{~g}$ and h). Interestingly, in the experiments with $\mathcal{D}_{\text {LTVC }}^{\text {quinary }}$ and $\mathcal{D}_{\text {AFLOW }}^{\text {quinary }}$, the numbers of quinary HEAs in the test set, and those found in the top 100 and top 1,000 HEA candidates recommended by the ERS, are much larger than those predicted by the competitor systems. These numbers are very high because the two data sets only contain quinary alloys of the early transition metals. Much of the evidence of the similarities between these element combinations can be collected from the corresponding data sets containing binary, ternary, and quaternary alloys (Figure $\mathrm{S} 1 \mathrm{~b}$ ). Moreover, to recall 50 and $75 \%$ of the quinary HEAs from these data sets, approximately 10-100 fewer trials are required by the ERS than by the NMF and SVD-based recommender systems. The results of experiments monitoring the dependence of the HEA recall ratio on the number of trials required are listed in detail in the Supplementary material (Section S VIB).

\section{E. Synthesis of recommended FeMnCo-based HEAs}

Fe-Co-based bulk soft-magnetic materials are widely applied to inductors, transformers, and electrical machines, and next-generation high-power devices will require such films to improve the performance of highfrequency and high-temperature devices ${ }^{33}$. Therefore, we focus on Fe-Co-based alloys containing the first transition-series elements. We apply the proposed ERS to recommend quaternary Fe-Co-based HEAs and experimentally validate them.

Figure 5 a shows the recommended possible magnetic quaternary HEAs containing Fe, Mn, and Co. Clearly, FeMnCoNi is the only HEA candidate recommended with a belief higher than 0.5. Although FeMnCoCr and FeM$\mathrm{nCoCu}$ are $\mathrm{HEA}$ candidates recommended with the next highest belief, some uncertainty still remains as to their potential as HEAs. Therefore, we chose FeMnCoNi as the target HEA candidate for the experimental validation (Figure 5, see the Supplementary material for further information).

Figure 5 c shows a 2D-XRD image of a region of the $\mathrm{Fe}_{0.25} \mathrm{Co}_{0.25} \mathrm{Mn}_{0.25} \mathrm{Ni}_{0.25}$ alloy annealed at $400^{\circ} \mathrm{C}$. A reflection attributed to the (110) plane of the BCC crystal structure appears in the ring pattern at $2 \theta=44.7^{\circ}$ (PDF 03-065-7519 $\left.9^{34}\right)$. Note that out-of-plane XRD measurements were also performed to identify the crystal structure in more detail, as shown in Figure $\mathrm{S} \mathrm{7(a)}$ in the Supplementary Information, indicating the formation of a polycrystalline film. Reportedly, the BCC crystal structure of the FeCoMn alloy is stable ${ }^{35}$, and there are no reports in the literature concerning the stability of the crystal structure of Ni-alloyed FeCoMn films. Therefore, to investigate the stability of the crystal structure, the effect of Ni doping on the crystal structure was analyzed based on the heat map generated from the X-ray diffraction patterns of FeCoMn films prepared with various $\mathrm{Ni}$ contents (Fig $5(\mathrm{~d})$ ). For an $\mathrm{Ni}$ content above 0.3 , the face-centered cubic (FCC) structure is also observed at 
$2 \theta=43.5^{\circ}$, corresponding to the $(111)$ reflection [Supplementary Figure S 7(b)] (PDF 03-065-513134). These results suggest that the $\mathrm{Fe}_{0.25} \mathrm{Co}_{0.25} \mathrm{Mn}_{0.25} \mathrm{Ni}_{0.25}$ HEA shows a BCC structure and is stable at high temperatures.

\section{CONCLUSIONS}

We present a descriptor-free ERS to recommend potential HEA candidates for a range of element combinations. In the experiments conducted using several data sets, the ERS shows the capability for measuring similarity, in terms of substitutability, between the element combinations. Furthermore, the ERS shows outstanding capability to recommend multicomponent alloys by extrapolation and learning from low-component alloy data, which is not exhibited by the competitor systems. In addition, the highly recommended Fe-Co-based magnetic HEA is experimentally validated by the proposed ERS; further, the results confirm that $\mathrm{FeCoMnNi}$ is stable at high temperatures and shows a BCC structure. In summary, these results corroborate that our work is promising for recommending HEAs and solving a wide range of combinatorial problems in materials science.

\section{ACKNOWLEDGMENTS}

This work is supported by the Ministry of Education, Culture, Sports, Science, and Technology of Japan (MEXT) ESICMM Grant Number 12016013; the Program for Promoting Research on the Supercomputer Fugaku (DPMSD); the JST-Mirai Program "Development of Materials Design Workflow and Data Library for Materials Foundry," Grant Number JPMJMI18G5; and JSPS KAKENHI Grants 20K05301, JP19H05815 (Grants-inAid for Scientific Research on Innovative Areas Interface Ionics), and 20K05068, Japan.

\section{DATA AVAILABILITY}

Data sets related to this article can be found at https://doi.org/10.31399/asm.hb.v03.a0006247, reference 20, and at https://doi.org/10.1016/j.actamat.2018.07.042, reference 23 .

\footnotetext{
${ }^{1}$ J. M. Rickman, H. M. Chan, M. P. Harmer, J. A. Smeltzer, C. J. Marvel, A. Roy, and G. Balasubramanian, "Materials informatics for the screening of multi-principal elements and high-entropy alloys," Nature Communications 10, 2618 (2019).

${ }^{2}$ M.-H. Tsai and J.-W. Yeh, "High-entropy alloys: A critical review," Materials Research Letters 2, 107-123 (2014), https://doi.org/10.1080/21663831.2014.912690.

${ }^{3}$ J.-W. Yeh, S.-K. Chen, S.-J. Lin, J.-Y. Gan, T.-S. Chin, T.-T. Shun, C.-H. Tsau, and S.-Y. Chang, "Nanostructured high-entropy alloys with multiple principal elements: Novel alloy design concepts and outcomes," Advanced Engineering Materials 6, 299-303 (2004), https://onlinelibrary.wiley.com/doi/pdf/10.1002/adem.200300567.
}

${ }^{4}$ B. Cantor, I. Chang, P. Knight, and A. Vincent, "Microstructural development in equiatomic multicomponent alloys," Materials Science and Engineering: A 375-377, 213 - 218 (2004).

${ }^{5}$ O. N. Senkov, J. D. Miller, D. B. Miracle, and C. Woodward, "Accelerated exploration of multi-principal element alloys with solid solution phases," Nature Communications 6, 6529 (2015).

${ }^{6}$ S. Gorsse, D. B. Miracle, and O. N. Senkov, "Mapping the world of complex concentrated alloys," Acta Materialia 135, 177 - 187 (2017).

${ }^{7}$ S. GUO and C. LIU, "Phase stability in high entropy alloys: Formation of solid-solution phase or amorphous phase," Progress in Natural Science: Materials International 21, 433 - 446 (2011). ${ }^{8}$ Y. Zhang, S. Guo, C. T. Liu, and X. Yang, "Phase formation rules," in High-Entropy Alloys: Fundamentals and Applications, edited by M. C. Gao, J.-W. Yeh, P. K. Liaw, and Y. Zhang (Springer International Publishing, Cham, 2016) pp. 21-49.

${ }^{9}$ W. P. Huhn and M. Widom, "Prediction of a2 to b2 phase transition in the high-entropy alloy mo-nb-ta-w," JOM 65, 1772-1779 (2013).

${ }^{10}$ M. Widom, "Prediction of structure and phase transformations," in High-Entropy Alloys: Fundamentals and Applications, edited by M. C. Gao, J.-W. Yeh, P. K. Liaw, and Y. Zhang (Springer International Publishing, Cham, 2016) pp. 267-298.

${ }^{11}$ D. D. Fontaine, "Cluster approach to order-disorder transformations in alloys," (Academic Press, 1994) pp. $33-176$.

${ }^{12}$ Y. Zhang, Y. Zhou, J. Lin, G. Chen, and P. Liaw, "Solid-solution phase formation rules for multi-component alloys," Advanced Engineering Materials 10, 534-538 (2008), https://onlinelibrary.wiley.com/doi/pdf/10.1002/adem.200700240.

${ }^{13}$ Y. Ye, Q. Wang, J. Lu, C. Liu, and Y. Yang, "Design of high entropy alloys: A single-parameter thermodynamic rule," Scripta Materialia 104, 53 - 55 (2015).

${ }^{14} \mathrm{M}$. Poletti and L. Battezzati, "Electronic and thermodynamic criteria for the occurrence of high entropy alloys in metallic systems," Acta Materialia 75, 297 - 306 (2014).

${ }^{15}$ E. P. George, D. Raabe, and R. O. Ritchie, "High-entropy alloys," Nature Reviews Materials 4, 515-534 (2019).

${ }^{16}$ A. P. Dempster, "Upper and lower probabilities induced by a multivalued mapping," The Annals of Mathematical Statistics 38, 325-339 (1967).

${ }^{17}$ A. P. Dempster, "A generalization of bayesian inference," Journal of the Royal Statistical Society: Series B (Methodological) 30, 205-232 (1968), https://rss.onlinelibrary.wiley.com/doi/pdf/10.1111/j.25176161.1968.tb00722.x.

${ }^{18}$ G. Shafer, A Mathematical Theory of Evidence (Princeton University Press, 1976).

${ }^{19}$ T. L. Fine, "Review: Glenn shafer, a mathematical theory of evidence," 83, 667-672 (1977).

${ }^{20}$ T. Denœux, D. Dubois, and H. Prade, "Representations of uncertainty in artificial intelligence: Beyond probability and possibility," in A Guided Tour of Artificial Intelligence Research, Vol. 1, edited by P. Marquis, O. Papini, and H. Prade (Springer Verlag, 2020) Chap. 4, pp. 119-150.

21 "Binary Alloy Phase Diagrams," in Alloy Phase Diagrams (ASM , International, 2016) https://dl.asminternational.org/chapterpdf/167711/a0006247.pdf.

${ }^{22}$ D. Alman, "Searching for next single-phase high-entropy alloy compositions," Entropy 15, 4504-4519 (2013).

${ }^{23}$ F. Zhang, C. Zhang, S. Chen, J. Zhu, W. Cao, and U. Kattner, "An understanding of high entropy alloys from phase diagram calculations," Calphad 45, 1 - 10 (2014).

${ }^{24}$ Y. Lederer, C. Toher, K. S. Vecchio, and S. Curtarolo, "The search for high entropy alloys: A high-throughput ab-initio approach," Acta Materialia 159, 364 - 383 (2018).

${ }^{25}$ A. Seko, H. Hayashi, H. Kashima, and I. Tanaka, "Matrix- and tensor-based recommender systems for the discovery of currently unknown inorganic compounds," Physical Review Materials 2, 013805 (2018). 
${ }^{26}$ P. Paatero, U. Tapper, P. Aalto, and M. Kulmala, "Matrix factorization methods for analysing diffusion battery data," Journal of Aerosol Science 22, S273 - S276 (1991), proceedings of the 1991 European Aerosol Conference.

${ }^{27}$ G. H. Golub and C. Reinsch, "Singular value decomposition and least squares solutions," Numer. Math. 14, 403-420 (1970).

${ }^{28}$ M. A. Hearst, "Support vector machines," IEEE Intelligent Systems 13, 18-28 (1998).

${ }^{29} \mathrm{P}$. McCullagh and J. Nelder, Generalized Linear Models, Second Edition, Chapman and Hall/CRC Monographs on Statistics and Applied Probability Series (Chapman \& Hall, 1989).

${ }^{30}$ J. R. Quinlan, "Induction of decision trees," Mach. Learn. 1, 81-106 (1986).

${ }^{31}$ G. I. Webb, "Naïve bayes," in Encyclopedia of Machine Learning, edited by C. Sammut and G. I. Webb (Springer US, Boston, MA,
2010) pp. $713-714$.

${ }^{32}$ F. Nielsen, "Hierarchical clustering," (2016) pp. 195-211.

${ }^{33}$ J. M. Silveyra, E. Ferrara, D. L. Huber, and T. C. Monson, "Soft magnetic materials for a sustainable and electrified world," Science 362 (2018), 10.1126/science.aao0195, https://science.sciencemag.org/content/362/6413/eaao0195.full.pdf.

${ }^{34} \mathrm{~S}$. Gates-Rector and T. Blanton, "The powder diffraction file: a quality materials characterization database," Powder Diffraction 34, 352-360 (2019).

${ }^{35}$ R. J. Snow, H. Bhatkar, A. T. N'Diaye, E. Arenholz, and Y. U. Idzerda, "Large moments in bcc fexcoymnz ternary alloy thin films," Applied Physics Letters 112, 072403 (2018). 
Figures

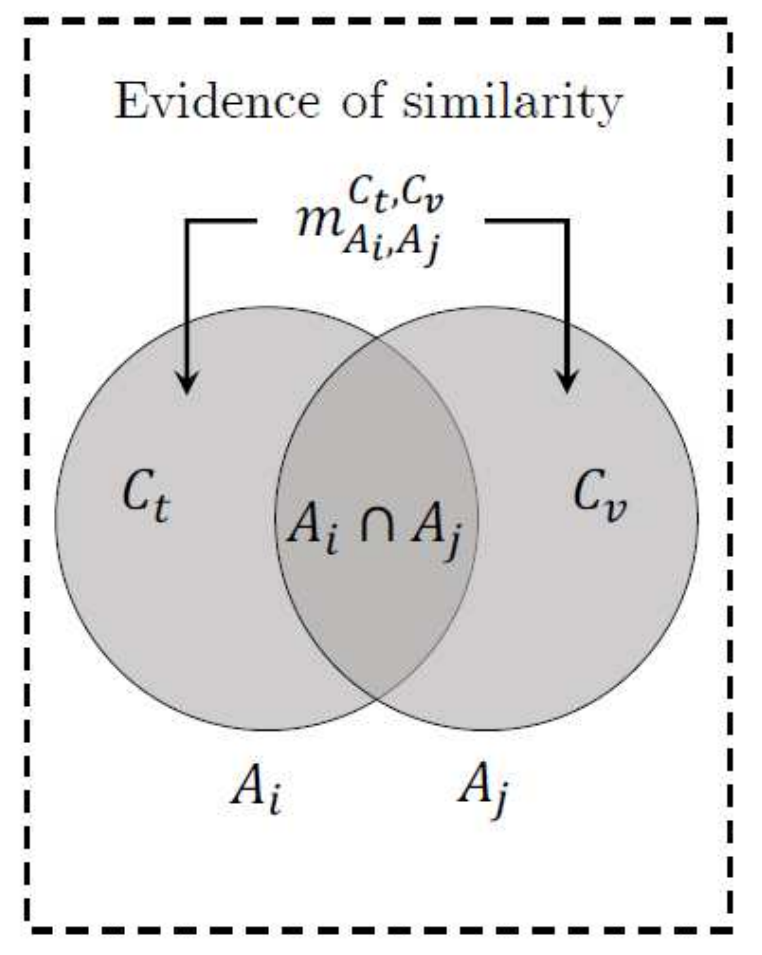

(a)

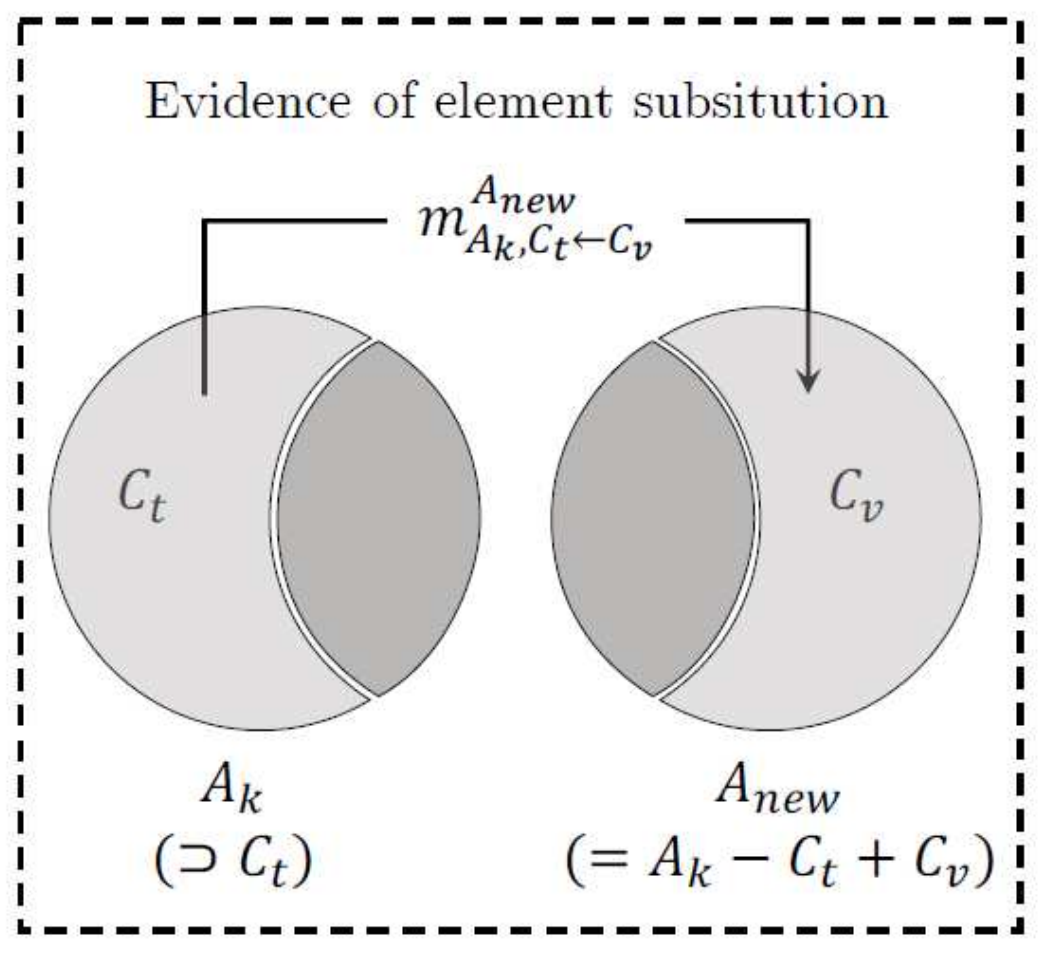

(b)

Figure 1

Schemes for using mass functions to model evidence of (a) similarities between element combinations and (b) new alloys by element substitution. 


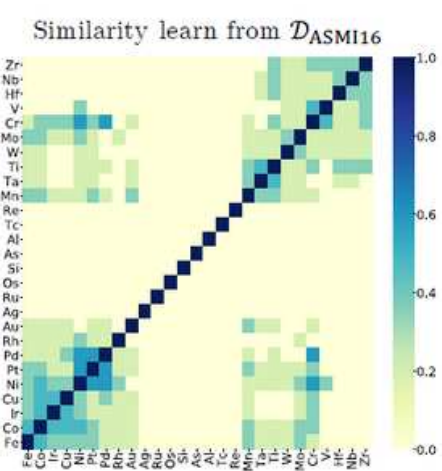

(a)

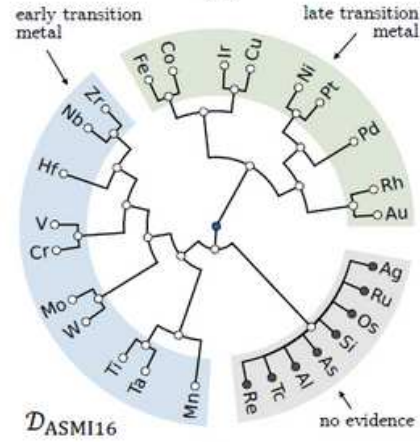

(e)

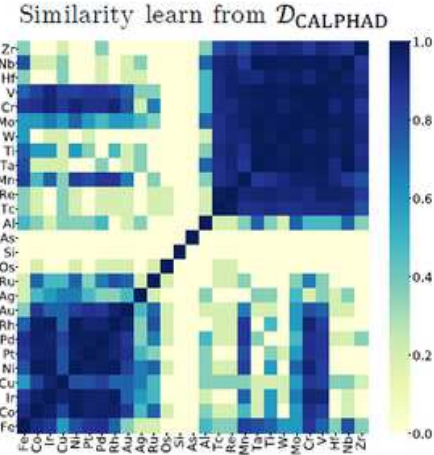

(b)

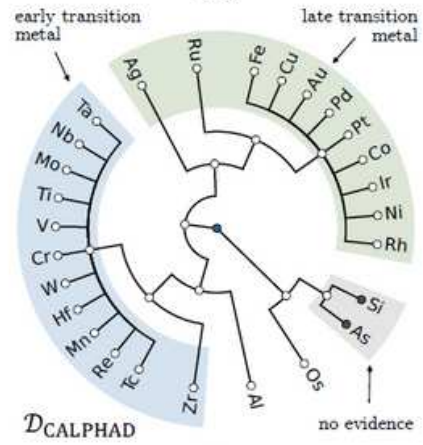

(f)

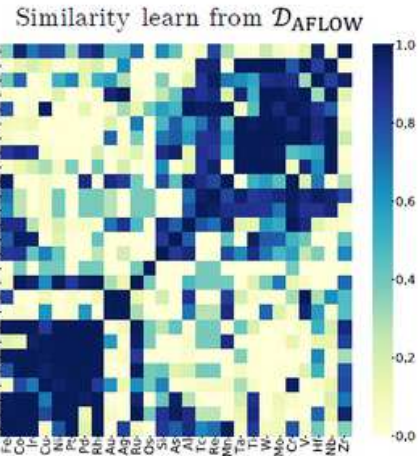

(c)

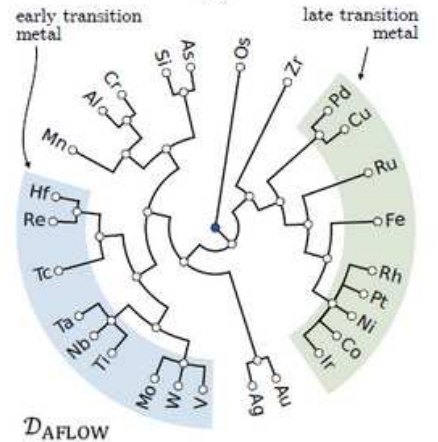

(g)
Similarity learn from $\mathcal{D}_{\text {LTVC }}$

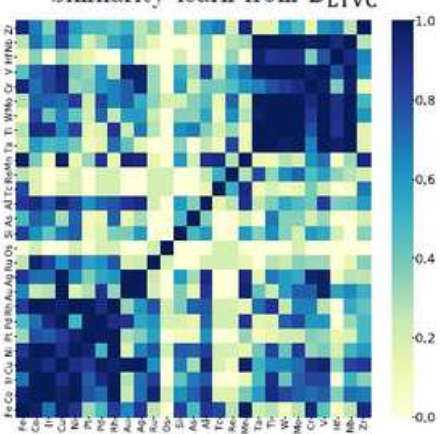

(d)

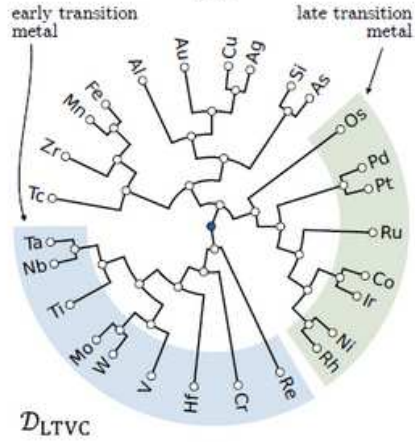

(h)

Figure 2

Top: Heat maps for similarity matrices among 27 elements E obtained from DASMI16, DCALPHAD, DAFLOW, and DLTVC data sets. Center: Hierarchically clustered structures of all elements in E constructed using these similarity matrices and hierarchical agglomerative clustering. The blue, green, and gray regions indicate groups of early and late transition metals, and elements without similarity evidence, respectively

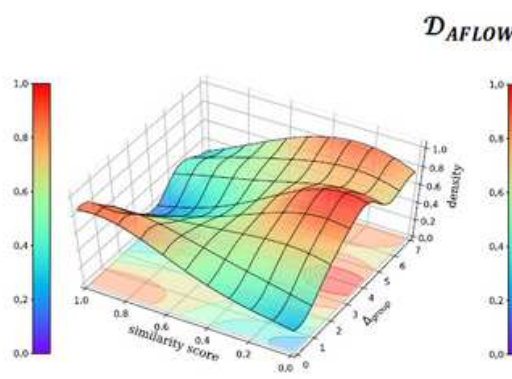

(a)

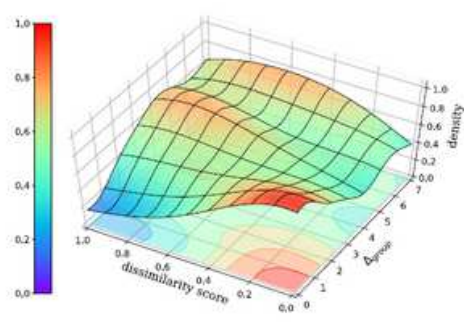

(b)

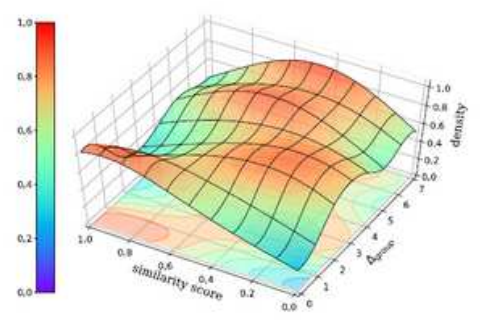

(c)
$\mathcal{D}_{\text {LTVC }}$

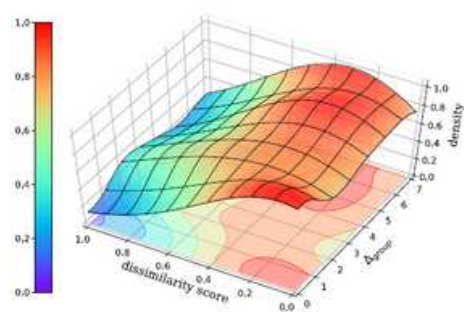

(d)

Figure 3

Correlation between pairwise similarity and difference in group index ( $\Delta$ group) for every element pair in $\mathrm{E}$, as learned from both data sets: DAFLOW $(i, j)$ and DLTVC $(k, l)$. 


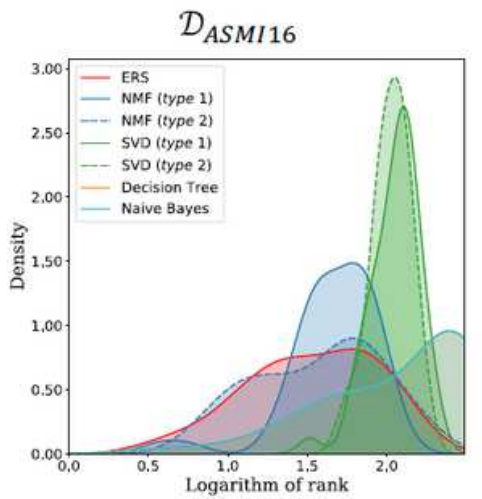

(a)

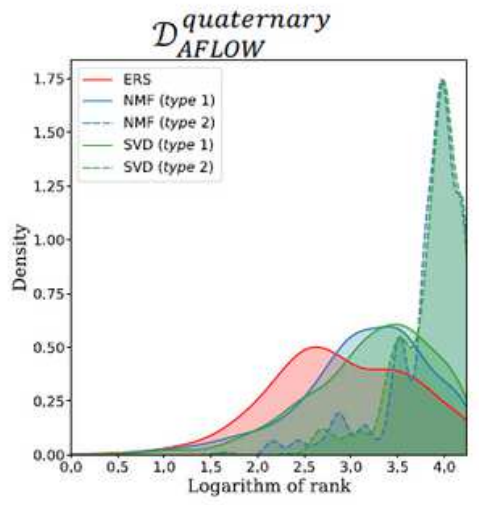

(e)

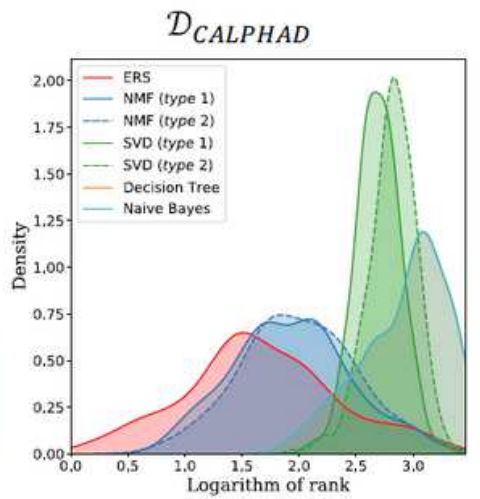

(b)

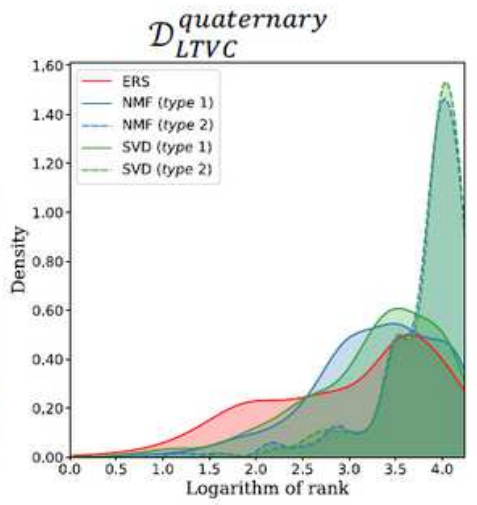

(f)

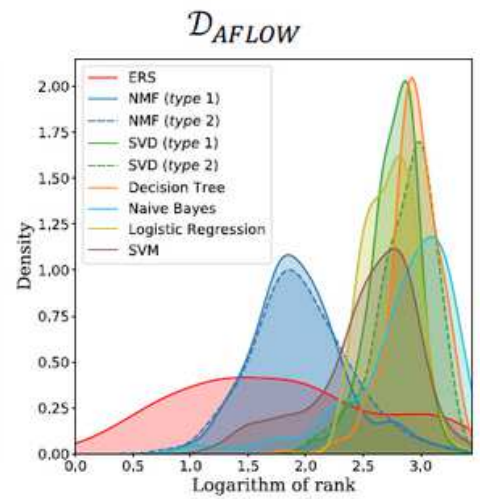

(c)

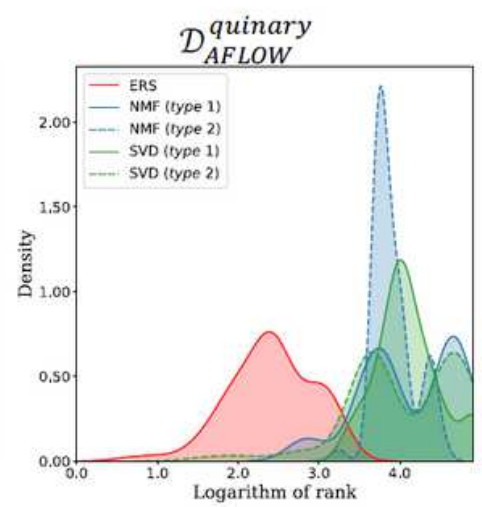

(g)

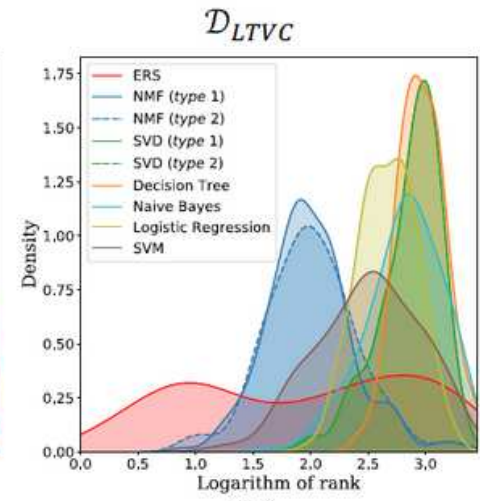

(d)

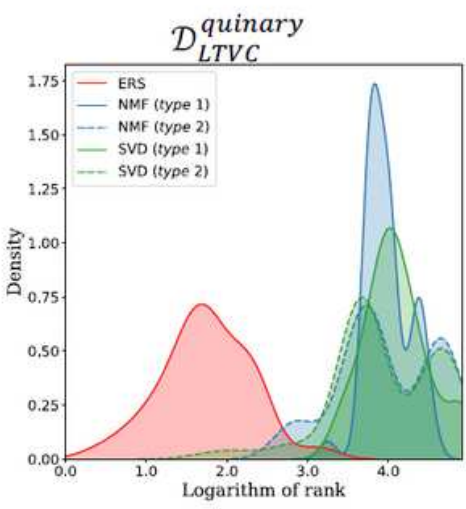

(h)

\section{Figure 4}

Probability density functions of the rank of the HEAs in the test sets in DASMI16, DCALPHAD, DAFLOW, DLTVC, Dquaternary AFLOW , Dquaternary LTVC , Dquinary AFLOW, and Dquinary LTVC experiments. The ranks of HEAs in the test sets are expressed on a base-10 logarithmic scale.

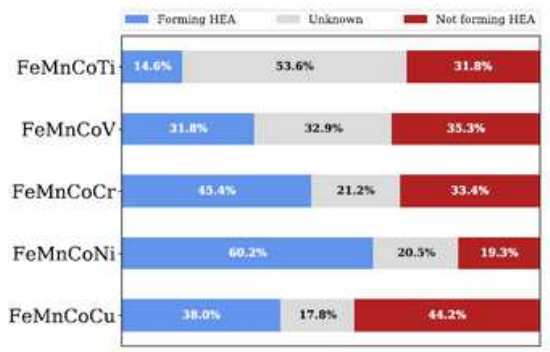

(a)

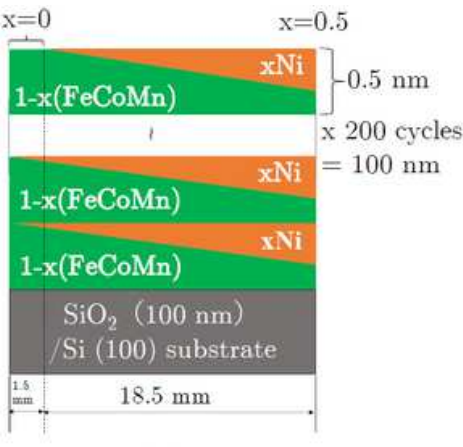

(b)

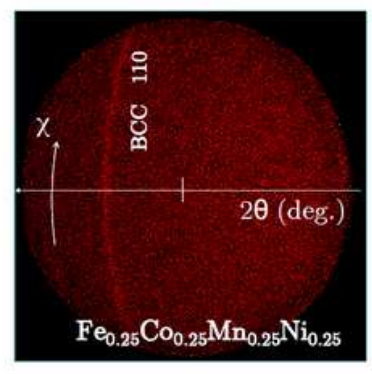

(c)

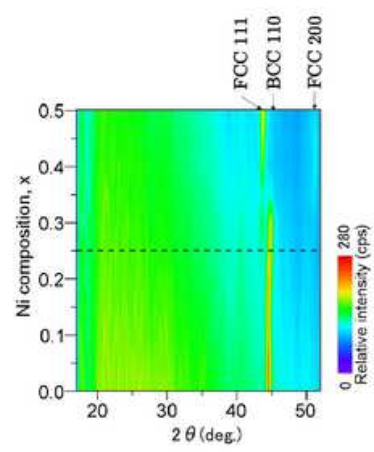

(d)

\section{Figure 5}

(a) Recommended candidates for Fe-Co-based HEAs containing first-transition-series elements:

FeMnCoTi, FeM-nCoV, FeMnCoCr, FeMnCoNi, and FeMnCoCu. (b) Schematic illustration of the sample. (c) 2D-XRD image of Fe0.25Co0.25Mn0.25Ni0.25 film. (d) Heat map of $\omega-2 \theta$ scan converted from 2D-XRD images of 1-x(FeCoMn)-xNi films pre-pared with various Ni contents. 


\section{Supplementary Files}

This is a list of supplementary files associated with this preprint. Click to download.

- supplementarymaterial.pdf 